De Tampere à Séville : bilan de la sécurité européenne $(2 / 2)$

\title{
Proposition de directive du parlement européen et du conseil concernant le traitement des données à caractère personnel et la protection de la vie privée dans le secteur des communications électroniques
}

Commission des communautés européennes

\section{OpenEdition \\ Journals}

\section{Édition électronique}

URL : http://journals.openedition.org/conflits/801

DOI : $10.4000 /$ conflits. 801

ISSN : 1777-5345

\section{Éditeur :}

CCLS - Centre d'études sur les conflits lilberté et sécurité, L'Harmattan

\section{Édition imprimée}

Date de publication : 1 juin 2002

ISBN : 2-7475-3030-2

ISSN : 1157-996X

Référence électronique

Commission des communautés européennes, «Proposition de directive du parlement européen et du conseil concernant le traitement des données à caractère personnel et la protection de la vie privée dans le secteur des communications électroniques », Cultures \& Conflits [En ligne], 46 | été 2002, mis en ligne le, consulté le 30 mars 2021. URL : http://journals.openedition.org/conflits/801 ; DOI : https:// doi.org/10.4000/conflits.801

Ce document a été généré automatiquement le 30 mars 2021.

Creative Commons License 


\section{Proposition de directive $\mathrm{du}$ parlement européen et du conseil concernant le traitement des données à caractère personnel et la protection de la vie privée dans le secteur des communications électroniques}

Commission des communautés européennes

1 EXPOSÉ DES MOTIFS ${ }^{1}$

2 1. INTRODUCTION

3 La directive proposée est destinée à remplacer la directive 97/66/CE concernant le traitement des données à caractère personnel et la protection de la vie privée dans le secteur des télécommunications, qui a été adoptée par le Parlement européen et le Conseil le 15 décembre 1997 et devait être transposée pour le 24 octobre 1998 au plus tard.

4 La proposition ne vise pas à modifier profondément le contenu de la directive existante, mais simplement à adapter et à actualiser ses dispositions pour tenir compte des évolutions récentes et prévisibles dans le domaine des services et des technologies des communications électroniques.

5 La majorité des dispositions de la directive existante sont donc intégrées dans la nouvelle directive moyennant des modifications rédactionnelles mineures.

6 2. BUTS ET OBJECTIFS

7 L'un des principes réglementaires exposés lors du réexamen 1999 du cadre réglementaire des services de communications électroniques est l'adoption de règles 
neutres sur le plan technologique, c'est-à-dire de règles qui n'imposent ni ne favorisent de manière discriminatoire l'utilisation d'un type de technologie particulier, mais font en sorte qu'un même service soit réglementé de manière équivalente, indépendamment des moyens mis en œuvre pour sa fourniture.

8 Ce principe implique également que les consommateurs et les utilisateurs jouissent du même niveau de protection, quelle que soit la technologie mise en œuvre pour la fourniture d'un service donné. Le maintien d'un niveau élevé de protection des données et de la vie privée de la population est l'un des buts déclarés du réexamen 1999 du cadre des télécommunications. 
1. Sauf indication contraire, les définitions figurant dans la directive 95/46/CE et dans la directive 2001/../CE du Parlement européen et du Conseil relative à un cadre réglementaire commun pour les réseaux et les services de communications électroniques s'appliquent aux fins de la présente directive. Les définitions suivantes sont également applicables. On entend par :

21 a) «utilisateur »: toute personne physique utilisant un service de communications électroniques accessible au public à des fins privées ou professionnelles sans être nécessairement abonnée à ce service ;

b) «données relatives au trafic » : toutes les données traitées au cours ou en vue de la transmission d'une communication dans un réseau de communications électroniques ; c) «données de localisation»: toutes les données traitées dans un réseau de communications électroniques indiquant la position géographique de l'équipement terminal d'un utilisateur d'un service de communications électroniques accessible au public ;

24 d) « communication » : toute information échangée ou transmise entre un nombre fini de parties au moyen d'un service de communications électroniques accessible au public ;

e) « appel » : une connexion établie au moyen d'un service téléphonique accessible au public permettant une communication bidirectionnelle en temps réel.

Article 3

Services concernés

27 1. La présente directive s'applique au traitement des données à caractère personnel dans le cadre de la fourniture de services de communications électroniques accessibles au public sur les réseaux publics de communications dans la Communauté.

28 2. Les articles 8,10 et 11 s'appliquent aux lignes d'abonnés connectées à des centraux numériques et, lorsque cela est techniquement possible et ne nécessite pas un effort économique disproportionné, aux lignes d'abonnés connectées à des centraux analogiques.

29 3. Lorsqu'il est techniquement impossible de se conformer aux exigences des articles 8 , 10 et 11 ou lorsque cela nécessite un investissement disproportionné, les Etats membres en informent la Commission.

\section{Article 4}

Sécurité

31 1. Le fournisseur d'un service de communications électroniques accessible au public doit prendre les mesures d'ordre technique et organisationnel appropriées afin de garantir la sécurité de ses services, le cas échéant conjointement avec le fournisseur du réseau public de communications en ce qui concerne la sécurité du réseau. Compte tenu des possibilités techniques les plus récentes et du coût de leur mise en œuvre, ces mesures garantissent un degré de sécurité adapté au risque existant.

32 2. Lorsqu'il existe un risque particulier de violation de la sécurité du réseau, le fournisseur d'un service de communications électroniques accessible au public doit informer les abonnés de ce risque ainsi que de tout moyen éventuel d'y remédier, y compris le coût que cela implique.

Article 5

Confidentialité des communications 
34 1. Les Etats membres garantissent, par la législation nationale, la confidentialité des communications effectuées au moyen d'un réseau public de communications et de services de communications électroniques accessibles au public, ainsi que la confidentialité des données relatives au trafic y afférentes. En particulier, ils interdisent à toute autre personne que les utilisateurs, sans le consentement des utilisateurs concernés, d'écouter, d'intercepter, de stocker les communications et les données relatives au trafic y afférentes, ou de les soumettre à tout autre moyen d'interception ou de surveillance, sauf lorsque cette personne y est légalement autorisée, conformément à l'article 15, paragraphe 1 .

2. Le paragraphe 1 n'affecte pas l'enregistrement légalement autorisé de communications et des données relatives au trafic y afférentes, dans le cadre des usages professionnels licites, afin de fournir la preuve d'une transaction commerciale ou de toute autre communication commerciale.

Article 6

Données relatives au trafic

37 1. Les données relatives au trafic concernant les abonnés et les utilisateurs traitées aux fins de la transmission d'une communication et stockées par le fournisseur d'un réseau ou d'un service public de communications doivent être effacées ou rendues anonymes dès l'achèvement de la transmission, sans préjudice des dispositions des paragraphes 2 , 3 et 4 .

38 2. Les données relatives au trafic qui sont nécessaires pour établir les factures des abonnés et les paiements pour interconnexion peuvent être traitées. Un tel traitement n'est autorisé que jusqu'à la fin de la période au cours de laquelle la facture peut être légalement contestée ou des poursuites engagées pour en obtenir le paiement.

39 3. Afin de commercialiser ses propres services de communications électroniques ou de fournir à l'abonné des services à valeur ajoutée, le fournisseur d'un service de communications électroniques accessible au public peut traiter les données visées au paragraphe 1 dans la mesure et pour la durée nécessaires à la fourniture de ces services, pour autant que l'abonné ait donné son consentement.

40 4. Le fournisseur de service doit informer l'abonné des types de données relatives au trafic qui sont traités aux fins visées aux paragraphes 2 et 3 et de durée de ce traitement.

41 5. Le traitement des données relatives au trafic effectué conformément aux dispositions des paragraphes 1 à 4 doit être restreint aux personnes, agissant sous l'autorité des fournisseurs de réseaux et de services de communications publics, chargées d'assurer la facturation ou la gestion du trafic, de répondre aux demandes de la clientèle, de détecter les fraudes et de commercialiser les services de communications électroniques du fournisseur ou de fournir un service à valeur ajoutée ; ce traitement doit se limiter à ce qui est nécessaire à de telles activités.

42 6. Les paragraphes $1,2,3$ et 5 s'appliquent sans préjudice de la possibilité qu'ont les autorités compétentes de se faire communiquer des données relatives au trafic conformément à la législation en vigueur dans le but de régler des litiges, notamment en matière d'interconnexion ou de facturation.

$43 \quad$ Article 7

Facturation détaillée 
1. Les abonnés ont le droit de recevoir des factures non détaillées.

2. Les Etats membres appliquent des dispositions nationales afin de concilier les droits des abonnés recevant des factures détaillées avec le droit à la vie privée des utilisateurs appelants et des abonnés appelés, par exemple en veillant à ce que lesdits utilisateurs et abonnés disposent de modalités complémentaires suffisantes renforçant le respect de la vie privée pour les communications ou les paiements.

\section{Article 8}

Présentation et restriction de l'identification de la ligne appelante et de la ligne connectée

1. Dans les cas où la présentation de l'identification de la ligne appelante est offerte, l'utilisateur appelant doit pouvoir empêcher, par un moyen simple et gratuit, la présentation de l'identification de la ligne appelante, et ce, appel par appel. L'abonné appelant doit avoir cette possibilité pour chaque ligne.

2. Dans les cas où la présentation de l'identification de la ligne appelante est offerte, l'abonné appelé doit pouvoir empêcher, par un moyen simple, gratuit pour un usage raisonnable de cette fonction, la présentation de l'identification de la ligne appelante pour les appels entrants.

3. Dans les cas où la présentation de l'identification de la ligne appelante est offerte et où l'identification de la ligne appelante est présentée avant l'établissement de l'appel, l'abonné appelé doit pouvoir, par un moyen simple, refuser les appels entrants lorsque l'utilisateur ou l'abonné appelant a empêché la présentation de l'identification de la ligne appelante.

4. Dans les cas où la présentation de l'identification de la ligne connectée est offerte, l'abonné appelé doit pouvoir, par un moyen simple et gratuit, empêcher la présentation de l'identification de la ligne connectée à l'utilisateur appelant.

1 5. Les dispositions du paragraphe 1 s'appliquent également aux appels provenant de la Communauté à destination de pays tiers. Les dispositions des paragraphes 2,3 et 4 s'appliquent également aux appels entrants provenant de pays tiers.

6. Les Etats membres veillent à ce que, dans les cas où la présentation de l'identification de la ligne appelante et/ou de la ligne connectée est offerte, les fournisseurs de services de communications électroniques accessibles au public informent le public de cette situation, ainsi que des possibilités prévues aux paragraphes 1 à 4 .

\section{Article 9}

Données de localisation

4 1. Lorsque les réseaux de communications électroniques sont en mesure de traiter des données de localisation autres que des données relatives au trafic, concernant des abonnés ou des utilisateurs de leurs services, ces données ne peuvent être traitées qu'après avoir été rendues anonymes ou avec le consentement des utilisateurs ou abonnés, dans la mesure et pour la durée nécessaires à la fourniture d'un service à valeur ajoutée. Le fournisseur du service doit informer les utilisateurs ou les abonnés, avant d'obtenir leur consentement, du type de données de localisation qui sera traité, des objectifs et de la durée de ce traitement, et du fait que les données seront ou non transmises à un tiers en vue de la fourniture du service à valeur ajoutée.

2. Lorsque les utilisateurs ou abonnés ont donné leur consentement au traitement des données de localisation autres que les données relatives au trafic, ils doivent garder la 
possibilité d'interdire temporairement, par un moyen simple et sans frais, le traitement de ces données pour chaque connexion au réseau ou pour chaque transmission de communication.

3. Le traitement des données de localisation effectué conformément aux dispositions des paragraphes 1 et 2 doit être restreint aux personnes agissant sous l'autorité du fournisseur du service de communications électroniques ou du tiers qui fournit le service à valeur ajoutée, et doit se limiter à ce qui est nécessaire pour assurer la fourniture du service à valeur ajoutée.

57 Article 10

Dérogations

58 Les Etats membres veillent à ce que des procédures transparentes régissent les modalités grâce auxquelles le fournisseur d'un réseau public de communications et/ou d'un service de communications électroniques accessible au public peut passer outre :

59 a) à la suppression de la présentation de l'identification de la ligne appelante, à titre temporaire, lorsqu'un abonné demande l'identification d'appels malveillants ou dérangeants; dans ce cas, conformément au droit interne, les données permettant d'identifier l'abonné appelant seront conservées et mises à disposition par le fournisseur d'un réseau public de communications et/ou d'un service de communications électroniques accessible au public ;

60 b) à la suppression de la présentation de l'identification de la ligne appelante et à l'interdiction temporaire ou à l'absence de consentement d'un abonné ou d'un utilisateur en ce qui concerne le traitement de données de localisation, ligne par ligne, pour les organismes chargés de traiter les appels d'urgence et reconnus comme tels par un Etat membre, y compris les services de police, les services d'ambulances et les pompiers, dans le but de réagir à de tels appels.

61 Article 11

Renvoi automatique d'appel

62 Les Etats membres veillent à ce que tout abonné ait la possibilité, par un moyen simple et sans frais, de mettre fin au renvoi automatique des appels par un tiers vers son terminal.

63 Article 12

Annuaires d'abonnés

64 1. Les Etats membres veillent à ce que les abonnés soient informés, gratuitement, des fins auxquelles est établi un annuaire d'abonnés imprimé ou électronique accessible au public ou consultable par l'intermédiaire de services de renseignements, dans lequel les données à caractère personnel les concernant peuvent figurer, ainsi que de toute autre possibilité d'utilisation reposant sur des fonctions de recherche intégrées dans les versions électroniques de l'annuaire.

65 2. Les Etats membres veillent à ce que les abonnés aient la possibilité de décider, gratuitement, si leurs données à caractère personnel, et lesquelles de ces données, doivent figurer dans des annuaires publics, dans la mesure où ces données sont pertinentes par rapport à la fonction des annuaires en question telle qu'elle a été établie par leur fournisseur. Ils font également en sorte que les abonnés puissent vérifier, corriger ou supprimer ces données. 
66 3. Les dispositions des paragraphes 1 et 2 s'appliquent aux abonnés qui sont des personnes physiques. Les Etats membres veillent également, dans le cadre du droit communautaire et des législations nationales applicables, à ce que les intérêts légitimes des abonnés autres que les personnes physiques soient suffisamment protégés en ce qui concerne leur inscription dans les annuaires publics.

Article 13

Communications non sollicitées

68 1. L'utilisation de systèmes automatisés d'appel sans intervention humaine (automates d'appel), de télécopieurs ou de courrier électronique à des fins de prospection directe ne peut être autorisée que si elle vise des abonnés ayant donné leur consentement préalable.

69 2. Les Etats membres prennent les mesures appropriées pour faire en sorte que, sans frais pour l'abonné, les communications non sollicitées par celui-ci et effectuées à des fins de prospection directe par d'autres moyens que ceux visés au paragraphe 1 ne soient pas autorisées, soit sans le consentement des abonnés concernés, soit à l'égard des abonnés qui ne souhaitent pas recevoir ces communications, le choix entre ces deux solutions étant régi par la législation nationale.

70 3. Les dispositions des paragraphes 1 et 2 s'appliquent aux abonnés qui sont des personnes physiques. Les Etats membres veillent également, dans le cadre du droit communautaire et des législations nationales applicables, à ce que les intérêts légitimes des abonnés autres que les personnes physiques soient suffisamment protégés en ce qui concerne les communications non sollicitées.

71 Article 14

Caractéristiques techniques et normalisation

72 1. Lors de la mise en œuvre des dispositions de la présente directive, les Etats membres veillent, sous réserve des paragraphes 2 et 3 , à ce qu'aucune exigence relative à des caractéristiques techniques spécifiques ne soit imposée aux terminaux ou à d'autres équipements de communications électroniques si elle risque d'entraver la mise sur le marché d'équipements ou la libre circulation de ces équipements dans les Etats membres et entre ces derniers.

2. Lorsque des dispositions de la présente directive ne peuvent être mises en œuvre qu'en imposant des caractéristiques techniques spécifiques aux réseaux de communications électroniques, les Etats membres en informent la Commission,

74 conformément aux procédures prévues par la directive 98/34/CE du Parlement européen et du Conseil du 22 juin 1998 prévoyant une procédure d'information dans le domaine des normes et réglementations techniques, modifiée par la directive 98/48/ CE9.

75 3. En tant que de besoin, la Commission adopte des mesures afin de garantir que les équipements terminaux comportent les sauvegardes nécessaires pour assurer la protection des données à caractère personnel et le respect de la vie privée des utilisateurs et des abonnés, conformément à la directive 1999/5/CE du 9 mars 1999 et à la décision 87/95/CEE du 22 décembre 1986 relative à la normalisation dans le domaine des technologies de l'information et des télécommunications.

76 Article 15

Application de certaines dispositions de la directive 951461CE 
77 1. Les Etats membres peuvent prendre des mesures législatives visant à limiter la portée des droits et des obligations prévus aux articles 5 et 6 , à l'article 8, paragraphes 1 à 4 et à l'article 9 lorsqu'une telle limitation constitue une mesure nécessaire pour sauvegarder la sûreté de l'Etat, la défense, la sécurité publique, la prévention, la recherche, la détection et la poursuite d'infractions pénales ou de l'utilisation non autorisée du système de communications électroniques, comme le prévoit l'article 13, paragraphe 1 , de la directive 95/46/CE.

78 2. Les dispositions du chapitre 111 de la directive 95/46/CE, relatif aux recours juridictionnels, à la responsabilité et aux sanctions, sont applicables aux dispositions nationales adoptées en application de la présente directive ainsi qu'aux droits individuels résultant de la présente directive.

79 3. Le groupe « protection des personnes à l'égard du traitement des données à caractère personnel » institué par l'article 29 de la directive 95/46/CE remplit aussi les tâches visées à l'article 30 de la directive $95 / 46 / C E$ en ce qui concerne les matières couvertes par la présente directive, à savoir la protection des droits et des libertés fondamentaux ainsi que des intérêts légitimes dans le secteur des communications électroniques.

\section{référence à la présente directive ou sont accompagnées d'une telle référence lors de leur publication officielle. Les modalités de cette référence sont arrêtées par les Etats membres.}

Les Etats membres communiquent à la Commission le texte des dispositions de droit interne qu'ils adoptent dans le domaine régi par la présente directive, ainsi que de toute modification ultérieure de ces dispositions. 


\section{NOTES}

1. Commission des communautés européennes, Bruxelles, le 12 juillet 2000 com(2000) 385 Voir également : position commune (CE) n²6/2002 du 28 janvier 2002 arrêtée par le Conseil (...) en vue de l'adoption d'une directive du Parlement européen et du Conseil concernant le traitement des données à caractère personnel et la protection de la vie privée dans le secteur des communications électroniques, Journal officiel $\mathrm{n}^{\circ} \mathrm{C} 113 \mathrm{E} \mathrm{du}$ 14/05/2002 p. 0039 - 0053.

\section{INDEX}

Mots-clés : droit européen, libertés publiques, textes et documents officiels, protection 